\title{
Perfil nUtRICIONAL E QUALIDADE DE VIDA DE CUIDADORES DE CRIANÇAS E ADOLESCENTES COM CÂNCER
}

\author{
NUTRITIONAL PROFILE AND QUALITY OF LIFE OF CAREGIVERS OF \\ CHILDREN AND ADOLESCENTS WITH CANCER
}

\author{
Juliana Zortéa ${ }^{1}$, Lethicia Lazzeri², Estela Beatriz Behling ${ }^{3}$, \\ Luciane Beitler da Cruz ${ }^{4}$, Lauro José Gregianin ${ }^{5}$
}

Clin Biomed Res. 2018;38(1):74-80

1 Pós-Graduação em Saúde da Criança e do Adolescente, Universidade Federal do Rio Grande do Sul (UFRGS). Porto Alegre, RS, Brasil.

2 Curso de Nutrição, Universidade Federal do Rio Grande do Sul (UFRGS). Porto Alegre, RS, Brasil.

3 Departamento de Nutrição, Faculdade de Medicina, Universidade Federal do Rio Grande do Sul (UFRGS). Porto Alegre, RS, Brasil.

4 Serviço de Nutrição, Hospital de Clínicas de Porto Alegre. Porto Alegre, RS, Brasil.

5 Pós-graduação em Saúde da Criança e do Adolescente, Departamento de Medicina, Faculdade de Medicina, Universidade Federal do Rio Grande do Sul (UFRGS). Porto Alegre, RS, Brasil.

Autor correspondente:

Juliana Zortéa

julizortea@hotmail.com

Pós-graduação em Saúde da Criança e do Adolescente, Universidade Federal do Rio Grande do Sul (UFRGS)

Rua Ramiro Barcelos, 2400.

90035-003, Porto Alegre, RS, Brasil.

\section{RESUMO}

Introdução: Verificar o perfil nutricional e a qualidade de vida dos cuidadores de crianças e/ou adolescentes com câncer durante os primeiros 6 meses de tratamento oncológico.

Métodos: Estudo de coorte realizado no período de julho/2015 a novembro/2016 em três momentos: até 2 semanas (T0) e em 3 (T1) e 6 (T2) meses após o diagnóstico. Foram aplicados um questionário sociodemográfico, um recordatório alimentar de 24 horas e o instrumento 36-Item Short-Form Health Survey (SF-36), e foi realizada a antropometria. Foram excluídos do estudo cuidadores gestantes, menores de 19 anos, responsáveis por pacientes em cuidados paliativos e em recidivas. A análise estatística utilizou o teste de dupla análise de variância de Friedman, e foram considerados significativos valores de $p<0,05$.

Resultados: Foram avaliados 42 cuidadores Os cuidadores mais frequentes foram mães (81\%), e a média de idade foi de 34,17 anos (DP $=8,94)$. Observou-se aumento no peso corporal, no índice de massa corporal, na circunferência abdominal e na dobra cutânea tricipital $(p<0,05)$. Foi constatada uma redução no consumo de calorias totais e de macronutrientes $(p<0,05)$, e o consumo de fibras ficou abaixo das recomendações. No aspecto qualidade de vida, o domínio vitalidade teve redução significativa $(p=0,042)$.

Conclusões: Os cuidadores de crianças e/ou adolescentes com câncer apresentam tendência a um consumo inadequado de alimentos. Os estados nutricionais predominantes foram o sobrepeso e a obesidade, com depósito de gordura visceral. Assim, o diagnóstico de câncer infantil interfere no estado nutricional e na qualidade de vida do cuidador durante os primeiros 6 meses do tratamento oncológico.

Palavras-chave: Câncer; cuidador; qualidade de vida; estado nutricional; criança; adolescente

\section{ABSTRACT}

Introduction: To assess the nutritional profile and quality of life of caregivers of children and adolescents with cancer during the first 6 months of cancer treatment.

Methods: A cohort study was conducted from July, 2015 to November, 2016 at three time points: up to 2 weeks (T0) and at 3 (T1) and 6 months (T2) after diagnosis. A sociodemographic questionnaire, a 24-hour recall, and a 36-Item Short-Form Health Survey (SF-36) questionnaire were applied, and anthropometry was performed. Pregnant caregivers, caregivers under 19 years old, and caregivers of patients in 
palliative care and of patients with recurrence were excluded from the study. The statistical analysis was performed using Friedman's two-way analysis of variance. $P$-value $<0.05$ was considered significant.

Results: We evaluated 42 caregivers. Mothers played the role of caregivers more frequently ( $81 \%)$, and mean age was 34.17 years $(S D=8.94)$. There was an increase in body weight, body mass index, waist circumference, and triceps skinfold thickness $(p<0.05)$. There was also a reduction in the consumption of total calories and macronutrients $(p<0.05)$, and fiber consumption was below recommendations. In terms of quality of life, vitality reduced significantly $(p=0.042)$.

Conclusion: Caregivers of children and/or adolescents with cancer tended to show inadequate food consumption. The prevailing nutritional states were overweight and obesity, with visceral fat deposit. Thus, the diagnosis of childhood cancer interferes in the nutritional status and quality of life of a caregiver during the first 6 months of cancer treatment.

Keywords: Cancer; caregiver; quality of life; nutritional status; children; adolescents

O câncer infantil é considerado uma gama de diferentes malignidades, e não uma única doença. Em geral, pouco se conhece sobre a etiologia do câncer na infância, principalmente por ser considerado raro em comparação ao câncer em adultos ${ }^{1}$.

Estima-se que a incidência dos tumores pediátricos no Brasil represente 3\% do total de casos de câncer, com estimativa de 12.600 novos casos para $2017^{2}$. Representa a primeira causa de morte $(7 \%$ do total) por doença entre crianças e adolescentes de 1 a 19 anos no Brasil ${ }^{3}$.

O diagnóstico inesperado de câncer, uma doença crônica potencialmente fatal, representa um grande desafio para o paciente e sua família. Uma mudança no cotidiano é imposta, fazendo-se necessário redimensionar as vidas dos envolvidos para conviver com a doença e suas implicações, que incluem dor, sofrimento e ameaça constante de terminalidade. Isso resulta em instabilidade familiar e extremo estresse para pais e/ou cuidadores ${ }^{4,5}$.

Crianças em geral necessitam de auxílio para as atividades de vida diária, como tomar banho, alimentar-se, vestir-se, entre outras. Quando se trata de crianças e adolescentes com câncer, essas necessidades se intensificam proporcionalmente à agressividade do tratamento. Esses cuidados intensivos tanto durante a internação hospitalar quanto nos períodos domiciliares podem trazer prejuízos a vários aspectos ligados à qualidade de vida $(\mathrm{QV})$ do cuidador, como sono, lazer, alimentação, autoestima, entre outros ${ }^{6-9}$.

No Brasil, não há disponibilidade de instrumentos validados e específicos para aferir a QV de cuidadores de crianças e adolescentes portadores de câncer ${ }^{8,10}$. Nesses casos, pode ser útil a aplicação de instrumentos genéricos, como o 36-Item Short-Form Health Survey (SF-36), que foi validado e adaptado culturalmente para a população brasileira em 1999. Esse questionário tem a vantagem de ser pouco extenso e de fácil compreensão e aplicação. É composto por 36 questões, aborda aspectos relacionados às últimas 4 semanas e tem o objetivo de avaliar a percepção individual de saúde e funcionamento ${ }^{11}$.

Devido às exigências da prestação de cuidados a crianças e adolescentes com câncer, os pais podem ser levados a negligenciar a sua própria saúde. Por isso, são necessárias pesquisas que analisem ações de autocuidado de promoção da saúde, incluindo qualidade do sono e alimentação.

O presente trabalho originou-se da necessidade de estudos abordando a QV no binômio paciente/cuidador, pois quanto melhor o cuidador estiver física e psicologicamente, melhor será o auxílio prestado ao paciente, o que contribuirá para o sucesso do tratamento oncológico. Além disso, estudos longitudinais sobre as modificações de QV são $\operatorname{raros}^{8,12}$ e abordam somente os 3 primeiros meses de tratamento.

O objetivo deste estudo foi verificar o estado nutricional, o consumo alimentar de energia e macronutrientes e a QV de cuidadores de crianças e adolescentes com câncer nos 6 primeiros meses de tratamento oncológico.

\section{MÉTODOS}

Trata-se de um estudo de coorte realizado no Serviço de Oncologia Pediátrica (SOP) do Hospital de Clínicas de Porto Alegre (HCPA), no período de julho de 2015 a novembro de 2016. Foram incluídos todos os cuidadores de novos casos de câncer infantojuvenil durante o período de estudo. As entrevistas ocorreram em três momentos: em até 2 semanas (T0), em 3 meses (T1) e em 6 meses (T2) após o diagnóstico. Houve um intervalo de tolerância de 10 dias antes ou após a data prevista para $\mathrm{T} 1 \mathrm{e}$ T2.

As entrevistas de T0 foram realizadas exclusivamente na unidade de internação do SOP do HCPA. Os questionários foram aplicados no quarto do paciente e, posteriormente, o cuidador 
foi encaminhado à sala de procedimentos para avaliação antropométrica. As entrevistas de T1 e T2 foram realizadas no ambulatório do SOP do HCPA, agendadas em horário anterior à consulta regular com o médico oncologista e aplicadas na sala de espera. As medidas antropométricas foram realizadas no consultório de nutrição. Nos casos em que o paciente estava internado, a entrevista seguiu o protocolo de T0.

Todos os participantes assinaram o termo de consentimento livre e esclarecido (TCLE). A pesquisa foi aprovada pelo Comitê de Ética e Pesquisa do HCPA, sob o parecer $n^{\circ} 1.121 .301$.

Os cuidadores responderam a um questionário sociodemográfico abordando questões como idade, sexo, escolaridade, grau de parentesco, cor, cidade de origem, estado marital, trabalho, presença de doenças crônicas, tabagismo e prática de atividade física.

O consumo alimentar foi verificado através da aplicação do recordatório alimentar de 24 horas (R24h). A partir dos dados coletados, foi calculado o consumo energético total (calorias) e a distribuição dos macronutrientes (proteína, lipídeos e carboidratos) em gramas e percentual sobre o valor energético total, utilizando o programa DietWin Plus ${ }^{\circledR}$, versão 2015. Os dados referentes à QV do cuidador foram obtidos pelo questionário autoaplicável SF-36 ${ }^{11}$.

A avaliação antropométrica dos cuidadores consistiu nas medidas de peso $(\mathrm{kg})$, estatura $(\mathrm{cm})$, dobra cutânea tricipital (DCT) $(\mathrm{mm})$ e circunferência abdominal (CA) (cm). Para avaliação do estado nutricional, foi calculado o índice de massa corporal (IMC), interpretado segundo os valores de referência da OMS para adultos: $<18,5 \mathrm{~kg} / \mathrm{m}^{2}=$ baixo peso; $\geq 18,5 \mathrm{a} 24,9 \mathrm{~kg} / \mathrm{m}^{2}=$ eutrófico; $\geq 24,9 \mathrm{a} 29,9 \mathrm{~kg} / \mathrm{m}^{2}=$ sobrepeso; $\geq 30$ a $34,9 \mathrm{~kg} / \mathrm{m}^{2}=$ obesidade $\mathrm{l} ; \geq 35$ a $40 \mathrm{~kg} / \mathrm{m}^{2}=$ obesidade II; e $>40 \mathrm{~kg} / \mathrm{m}^{2}=$ obesidade $\mathrm{III}{ }^{13}$. A CA foi verificada aplicando-se uma fita retrátil na maior circunferência entre a última costela e o ilíaco ao final de uma expiração ${ }^{14}$. Foram considerados em risco para doenças cardiovasculares mulheres com valores de $C A \geq 80 \mathrm{~cm}$ e homens $\geq 94 \mathrm{~cm}^{15}$. Para avaliação do estado nutricional segundo a DCT, foram utilizados os critérios estabelecidos por Frisancho ${ }^{13}$.

\section{Análise Estatística}

A análise dos dados foi feita utilizando o software SPSS ${ }^{\circledR}$, versão 21.0. Foram analisadas as possíveis alterações nas variáveis ao longo do tempo (T0, T1 e T2). Todos os dados foram testados quanto às características de distribuição pelo teste de normalidade de Shapiro-Wilk. Para variáveis não paramétricas, foi utilizado o teste de dupla análise de variância de Friedman de amostras relacionadas por postos. As variáveis paramétricas foram testadas por equações de estimativas generalizadas. A estatística descritiva foi utilizada para caracterização demográfica dos participantes. Os resultados foram considerados significativos a um nível de significância máximo de $5 \%(p<0,05)$.

\section{RESULTADOS}

Foram convidados a participar do estudo 58 cuidadores de crianças e adolescentes com câncer. Desses, dois não concordaram em assinar o TCLE e uma declarou ser gestante, restando 55 sujeitos no T0. No T2, foram entrevistados 42 cuidadores; portanto, houve uma perda de $24 \%$ da amostra ao longo do tempo, devido principalmente a óbitos de pacientes e abandono do tratamento por parte do cuidador. A Figura 1 mostra a formação do grupo em estudo.

\section{Motivos Para Exclusões}

Convidados a participar

1: gestante

2: não aceitaram

4: óbito do paciente

3: não compareceram dentro do tempo limite

1: desistência

1: parou de acompanhar o tratamento

1: óbito do paciente

1: desistência

1: não compareceu dentro do tempo limite

1: parou de acompanhar o tratamento

Figura 1: Formação do grupo de estudo. 
A amostra foi composta predominantemente pelo sexo feminino $(88,1 \%)$, sendo os cuidadores preferenciais as mães $(81 \%)$, seguidas dos pais $(9,5 \%)$ e outros $(9,5 \%)$. A média de idade foi 34,17 anos (DP $=8,94)$, variando de 19 a 58 anos. Quanto ao nível de escolaridade, $73,8 \%$ haviam estudado menos de 11 anos, ou seja, possuíam ensino médio incompleto. Observou-se um aumento no número de desempregados de 9,09\% (T0) para 59,5\% (T2), assim como uma redução da prática de atividades físicas de 30,9\% (T0) para 16,7\% (T2). A maior parte da amostra era procedente da região metropolitana de Porto Alegre $(52,4 \%)$, enquanto $47,6 \%$ residiam no interior do estado do Rio Grande do Sul (Tabela 1).
A análise dos parâmetros antropométricos identificou aumentos significativos no peso, IMC, CA e DCT dos cuidadores (Tabela 2). De acordo com a classificação de IMC, no T0, $58,18 \%$ dos cuidadores foram classificados com excesso de peso (sobrepeso e/ou obesidade) e, no T2, esse número aumentou para $61,9 \%$ (Tabela 3). No T0, não havia indivíduos com obesidade grau III e, no T2, eram $2,38 \%$, dado que vem ao encontro dos dados obtidos na DCT, em que $43,73 \%$ (T0) e $76,19 \%$ (T3) foram classificados como obesos.

No T0, $73,8 \%$ dos cuidadores foram considerados "em risco" para doença cardiovascular de acordo com os critérios de CA e, no T3, o percentual aumentou para $78,57 \%$. Na avaliação do consumo alimentar,

Tabela 1: Caracterização da amostra.

\begin{tabular}{|c|c|c|c|}
\hline Características & T0 (N = 55) & $\mathrm{T} 1(\mathrm{~N}=46)$ & $\mathrm{T} 2(\mathrm{~N}=42)$ \\
\hline Idade - Média \pm Desvio padrão & $34,69 \pm 9,86$ & $33,59 \pm 8,88$ & $34,17 \pm 8,94$ \\
\hline \multicolumn{4}{|l|}{ Sexo $-\mathrm{n}(\%)$} \\
\hline Feminino & $49(89,1)$ & $40(86,95)$ & $37(88,1)$ \\
\hline Masculino & $6(10,9)$ & $6(13,05)$ & $5(11,9)$ \\
\hline \multicolumn{4}{|l|}{ Cor - n (\%) } \\
\hline Branca & $39(70,1)$ & $32(69,5)$ & $29(69)$ \\
\hline Não branca & $16(29,09)$ & $14(30,5)$ & $13(31)$ \\
\hline \multicolumn{4}{|l|}{ Escolaridade - n (\%) } \\
\hline Analfabeto & $1(1,81)$ & $1(2,17)$ & 0 \\
\hline Fundamental incompleto & $21(38,18)$ & $18(39,1)$ & $17(40,48)$ \\
\hline Fundamental completo & $9(16,36 \%)$ & $7(15,2)$ & $7(16,7 \%)$ \\
\hline Médio incompleto & $8(14,54 \%)$ & $8(17,39)$ & $7(16,7 \%)$ \\
\hline Médio completo & $8(14,54 \%)$ & $7(15,2)$ & $6(14,3 \%)$ \\
\hline Graduação incompleta & $2(3,63 \%)$ & $2(4,34)$ & $2(4,8 \%)$ \\
\hline Graduação completa & $5(9,09 \%)$ & $2(4,34)$ & $2(4,8 \%)$ \\
\hline Pós-graduação & $1(1,81 \%)$ & $1(2,17)$ & $1(2,4 \%)$ \\
\hline \multicolumn{4}{|l|}{ Doença crônica } \\
\hline Sim - n (\%) & $14(25,45)$ & $11(23,91)$ & $9(21,4)$ \\
\hline Não - n (\%) & $41(74,54)$ & $35(76,09)$ & $33(78,6)$ \\
\hline \multicolumn{4}{|l|}{ Estado marital - n (\%) } \\
\hline Solteiro & $7(12,72)$ & $6(13,04)$ & $6(14,3)$ \\
\hline Mora junto/casado & $40(72,72)$ & $33(71,73)$ & $25(59,5)$ \\
\hline Divorciado & $4(7,27)$ & $4(8,7)$ & $8(19)$ \\
\hline Viúvo & $4(7,27)$ & $3(6,5)$ & $3(7,1)$ \\
\hline \multicolumn{4}{|l|}{ Tabagismo } \\
\hline Sim - n (\%) & $15(27,27 \%)$ & $14(30,5)$ & $10(23,8 \%)$ \\
\hline Não - n (\%) & $40(72,72)$ & $32(69,5)$ & $32(76,2)$ \\
\hline \multicolumn{4}{|l|}{ Atividade física } \\
\hline Sim - n (\%) & $17(30,9)$ & $12(26,1)$ & $7(16,7)$ \\
\hline Não - n (\%) & $38(69,1)$ & $34(73,9)$ & $35(83,3)$ \\
\hline \multicolumn{4}{|l|}{ Trabalho } \\
\hline Remunerado - n (\%) & $39(70,9)$ & $25(54,34)$ & $8(19)$ \\
\hline Desempregado - n (\%) & $5(9,09)$ & $12(26,1)$ & $25(59,5)$ \\
\hline Aposentado - n (\%) & $3(5,45)$ & $2(4,34)$ & $2(4,8)$ \\
\hline Do lar - n (\%) & $8(14,54)$ & $7(15,21)$ & $7(16,7)$ \\
\hline
\end{tabular}


Tabela 2: Características antropométricas, nutricionais e domínios de qualidade de vida (SF-36) dos cuidadores nos três momentos do estudo.

\begin{tabular}{|c|c|c|c|c|}
\hline Variáveis & T0 & T1 & T2 & $p$ \\
\hline Antropométricas & & Mediana (P25 - P75) & & \\
\hline $\mathrm{CA}(\mathrm{cm})$ & $90(79,12-99)^{\mathrm{a} *}$ & $89,5(78,75-103,5)^{\mathrm{ab}}$ & $93(81-102,70)^{b}$ & 0,029 \\
\hline $\mathrm{DCT}(\mathrm{mm})$ & $25(19,75-30,5)^{a}$ & $26,5(20,75-33)^{a}$ & $31(25-35)^{b}$ & 0,001 \\
\hline Peso $(\mathrm{kg})$ & $67,75(54,63-83,23)^{a}$ & $68,05(56,33-84,40)^{\mathrm{b}}$ & $69,65(57,72-83,17)^{\mathrm{b}}$ & $<0,001$ \\
\hline $\mathrm{IMC}\left(\mathrm{kg} / \mathrm{m}^{2}\right)$ & $26,10(22,36-32,24)^{a}$ & $26,27(22,72-32,01)^{b}$ & $26,19(22,58-32,83)^{b}$ & 0,009 \\
\hline Nutricionais & & Mediana (P25 - P75) & & \\
\hline Calorias (kcal) & $1701,43(1399,42-1940,72)^{a}$ & $1488,03(1153,04-2003,59)^{a}$ & $1282,49(918,28-1676,35)^{b}$ & 0,009 \\
\hline Carboidratos (g) & $210,44(159,87-267,99)^{a}$ & $183,54(140,07-261,75)^{\mathrm{a}}$ & $171,75(110,76-228,81)^{a}$ & 0,049 \\
\hline Fibras (g) & $16,58(13,38-22,80)^{a}$ & $13,35(7,14-17,74)^{\mathrm{b}}$ & $9,69(5,55-15,62)^{\mathrm{b}}$ & $<0,001$ \\
\hline Proteínas (g) & $81,47(67,39-101,52)^{a}$ & $68,85(43,24-93,91)^{\mathrm{b}}$ & $58,77(38,64-74,91)^{b}$ & $<0,001$ \\
\hline Lipídeos (g) & $55,30(42,91-69,75)^{a}$ & $51,92(37,43-66,53)^{a}$ & $38,10(30,90-52,52)^{b}$ & $<0,001$ \\
\hline Domínios do SF-36 & & Média \pm DP & & \\
\hline Capacidade funcional & $84,88(18,22)$ & $83,69(20,92)$ & $79,64(24,37)$ & 0,439 \\
\hline $\begin{array}{l}\text { Limitações por } \\
\text { aspectos físicos }\end{array}$ & $67,26(38,44)$ & $66,07(38,18)$ & $58,92(43,76)$ & 0,202 \\
\hline Dor & $70,40(20,47)$ & $66,85(24,86)$ & $69,76(23,51)$ & 0,812 \\
\hline $\begin{array}{l}\text { Estado de saúde } \\
\text { geral }\end{array}$ & $62,02(16,02)$ & $58(14,90)$ & $59,69(15,93)$ & 0,173 \\
\hline Vitalidade & $66,30(20,54)^{a}$ & $64,52(23,29)^{a}$ & $58,92(24,63)^{b}$ & 0,042 \\
\hline $\begin{array}{l}\text { Limitações por } \\
\text { aspectos sociais }\end{array}$ & $57,73(26,32)$ & $61,90(32,30)$ & $61,04(27,48)$ & 0,613 \\
\hline $\begin{array}{l}\text { Limitações por } \\
\text { aspectos emocionais }\end{array}$ & $44,44(45,17)$ & $62,68(43,06)$ & $50(42,45)$ & 0,096 \\
\hline Saúde mental & $61,52(20,58)$ & $66,38(26,13)$ & $61,71(23,70)$ & 0,224 \\
\hline
\end{tabular}

CA: circunferência abdominal; DCT: dobra cutânea tricipital; IMC: índice de massa corporal; *Letras diferentes representam resultado significativo.

Tabela 3: Classificação do estado nutricional da amostra nos três momentos do estudo.

\begin{tabular}{lccc}
\hline \multicolumn{1}{c}{ Variáveis } & T0 - $\mathbf{n}(\%)$ & T1 - $\mathbf{n ~ ( \% ) ~}$ & T2 - $\mathbf{n}(\%)$ \\
\hline IMC & & & $1(2,38)$ \\
Baixo peso & $2(3,63)$ & $1(2,17)$ & $15(35,71)$ \\
Eutrofia & $21(38,18)$ & $18(39,13)$ & $13(30,95)$ \\
Sobrepeso & $15(27,27)$ & $13(23,63)$ & $9(21,42)$ \\
Obesidade grau I & $13(23,63)$ & $10(21,73)$ & $3(7,14)$ \\
Obesidade grau II & $4(7,27)$ & $4(8,69)$ & $1(2,38)$ \\
Obesidade grau III & 0 & 0 & $4(9,52)$ \\
DCT & $10(18,18)$ & $3(7,14)$ \\
Desnutrição & $12(21,81)$ & $6(13,04)$ & $3(7,14)$ \\
Eutrofia & $9(16,36)$ & $10(21,73)$ & $32(76,19)$ \\
Sobrepeso & $24(43,63)$ & $3(6,52)$ & $27(58,69)$ \\
Obesidade & &
\end{tabular}

foi constatada uma redução significativa no consumo calórico total e em gramas de macronutrientes ao longo do tempo $(p<0,05)$ (Tabela 2). Porém, foram verificados valores crescentes na proporção de carboidratos (de 49,82\% para $54,67 \%$ em T0 e T2, respectivamente). Considerando-se a Recommended Dietary Allowances (RDA) de $25 \mathrm{~g} / \mathrm{dia}$ para mulheres e $38 \mathrm{~g} /$ dia para homens, o consumo de fibras ficou abaixo do recomendado nos três momentos do estudo (Tabela 2$)^{16}$.
No aspecto $Q V$, o domínio vitalidade foi o único que apresentou redução significativa ao longo do tempo de tratamento oncológico da criança ou adolescente $(p=0,042)$ (Tabela 2$)$.

\section{DISCUSSÃO}

Nossa amostra foi constituída prioritariamente por mães (81\%), que, segundo Wysocki et al. (2004), tendem a estar mais envolvidas com o cuidado da 
criança doente e mais propensas a permanecer no hospital ${ }^{17}$.

O excesso de peso destacou-se na avaliação antropométrica, com 58,18\% (T0) e 61,9\% (T2), resultado que vem ao encontro de dados do Instituto Brasileiro de Geografia e Estatística (IBGE) ${ }^{18}$ para a região Sul do Brasil, onde essa população corresponde a 50\%. O aumento significativo de peso ocorreu mesmo com a redução relatada do consumo calórico. Resultado semelhante foi apresentado por Smith et al. ${ }^{12}$, que mostrou que pais de crianças diagnosticadas com câncer apresentaram maior ganho ponderal e menor ingestão calórica ao longo dos 3 primeiros meses de tratamento.

Com relação à ingestão alimentar, houve redução significativa da ingestão de calorias totais e macronutrientes e um consumo percentual crescente de carboidratos (Tabela 2). Esse fato, associado ao baixo consumo de fibras, remete a uma alimentação rica em carboidratos simples, que predispõem a um risco aumentado de obesidade, doenças cardiovasculares e diabetes ${ }^{19}$ tipo 2. Essa mesma tendência de consumo foi encontrada na Pesquisa de Orçamento Familiar (POF 2008-2009), que identificou uma ingestão média de $12,5 \mathrm{~g}$ de fibras diárias e o açúcar simples contribuindo com $14,1 \%$ do consumo calórico total, enquanto o recomendado pela OMS é que não ultrapasse $10 \%{ }^{18}$. Além disso, sabe-se que a ingestão adequada de fibras pode minimizar o problema de doenças cardiovasculares devido à redução do colesterol total e da LDL plasmáticos ${ }^{20}$.

A avaliação antropométrica revelou uma prevalência de sobrepeso e obesidade, com tendência a depósito de gordura visceral, fator importante para a morbimortalidade e o risco de doenças cardiovasculares. Essa informação é relevante, pois justifica a aplicação de condutas preventivas e educativas a essa população. Essa intervenção pode determinar um benefício direto ao paciente que é cuidado por um indivíduo nas suas melhores condições de saúde. A obesidade com predomínio de gordura na região abdominal associa-se, com frequência, à dislipidemia, à resistência à insulina e à hipertensão arterial, aumentando ainda mais as chances de complicações cardiovasculares ${ }^{21}$.

$\mathrm{Na}$ avaliação da $\mathrm{QV}$, o único domínio em que houve variação significativa foi o de vitalidade, com uma queda no índice de 66,3 para 58,9 (T0 para T2) $(p<0,042)$. Nos demais domínios, os valores no T0 foram similares aos escores observados em outros estudos de metodologia transversal que compararam a QV de cuidadores ou pais de crianças com câncer com cuidadores ou pais de crianças saudáveis ${ }^{7,8,22-24}$. Esses dados sugerem que, já no momento do diagnóstico, existe um impacto nos domínios de QV, e não somente depois de instituído o tratamento, como sugerido por Klassen et al. ${ }^{9}$.

Este estudo concluiu que os cuidadores de crianças e/ou adolescentes com câncer apresentam um consumo alimentar rico em carboidratos e pobre em fibras nos 6 primeiros meses de tratamento. O diagnóstico de câncer infantil interfere imediatamente na QV do cuidador, que tende a permanecer comprometida durante o tratamento oncológico. Houve prevalência de indivíduos com sobrepeso e obesidade, e tendência a depósito de gordura visceral, sugerindo que orientações preventivas sobre ingestão alimentar e QV possam repercutir positivamente nos cuidados do paciente.

Este estudo apresentou limitações em sua metodologia, principalmente na utilização do R24h, que tem desvantagens se comparado a outros métodos, como depender da memória do entrevistado.

\section{Conflito de interesse}

Os autores declaram não ter conflitos de interesse.

\section{REFERÊNCIAS}

1. Braga PE, Latorre MR, Curado MP. Câncer na infância: análise comparativa da incidência, mortalidade e sobrevida em Goiânia (Brasil) e outros países. Cad Saude Publica. 2002;18:33-44.

2. Instituto Nacional de Câncer José Alencar Gomes da Silva (INCA). Tipos de câncer infantil. Rio de Janeiro: INCA; 2016. [citado 2016 Dez 18]. Disponível em: http://www2.inca.gov. $\mathrm{br} / \mathrm{wps} / \mathrm{wcm} /$ connect/tiposdecancer/ site/home/infantil
3. Instituto Nacional de Câncer José Alencar Gomes da Silva (INCA). Estimativa 2014: incidência de câncer no Brasil. Rio de Janeiro: INCA; 2014.

4. Motta MG. O entrelaçar dos mundos: família e hospital. In: Elsen I, Marcon SS, Santos MR, editores. O viver em familia e sua interface com a saúde e a doença. Maringá: EDUEM; 2002. p. 159-79.

5. Nascimento LC, Rocha SM, Hayes VH, Lima RA. Crianças com câncer e suas famílias. Rev Esc Enferm USP. 2005;39:469-74.
6. Cruz DA, Pimenta CA, Kurita GP, Oliveira AC, Boisvert C, Kamitsuru $\mathrm{S}$, et al. Caregivers of patients with chronic pain: responses to care. Int $J$ Nurs Terminol Classif. 2004;15:5-14.

7. Yamazaki S, Sokejima S, Mizoue T, Eboshida A, Fukuhara S. Healthrelated quality of life of mothers of children with leukemia in Japan. Qual Life Res. 2005;14:1079-85.

8. Goldbeck L. The impact of newly diagnosed chronic paediatric conditions on parental quality of life. Qual Life Res. 2006;15:1121-31. 
9. Klassen AF, Klassen R, Dix D, Pritchard S, Yanofsky R, O'Donnell $M$, et al. Impact of caring for a child with cancer on parents' healthrelated quality of life. J Clin Oncol. 2008;26:5884-9.

10. Wells DK, James K, Stewart JL, Moore IM, Kelly KP, Moore B, et al. The care of my child with cancer: a new instrument to measure caregiving demand in parents of children with cancer. J Pediatr Nurs. 2002;17:20110.

11. Ciconelli RM, Ferraz MB, Santos W, Meinão I, Quaresma MR. Tradução para a língua portuguesa e validação do questionário genérico de avaliação de qualidade de vida SF-36 (Brasil SF-36). Rev Bras Reumatol. 1999;39:143-50.

12. Smith AW, Baum A, Wing RR. Stress and weight gain in parents of cancer patients. Int J Obes. 2005;29:244-50.

13. World Health Organization (WHO). Physical status: the use and interpretation of anthropometry. Geneva: WHO; 1995.
14. Cole TJ. The LMS method for constructing normalized growth standards. Eur J Clin Nutr. 1990;44:45-60.

15. Associação Brasileira para o Estudo da Obesidade e da Síndrome Metabólica (ABESO). Diretrizes brasileiras de obesidade 2009/2010. 3. ed. Itapevi: AC Farmacêutica; 2009.

16. Food and Nutrition Board. Institute of Medicine (U.S.). Dietary reference intakes for energy, carbohydrate, fiber, fat, fatty acids, choresterol, protein, and amino acids. Washington: The National Academies Press; 2005.

17. Wysocki T, Gavin L. Psychometric properties of a new measure of fathers' involvement in the management of pediatric chronic diseases. J Pediatr Psychol. 2004;29:231-40.

18. Instituto Brasileiro de Geografia e Estatística (IBGE). Pesquisa de Orçamentos Familiares 2008-2009: antropometria e estado nutricional de crianças, adolescentes e adultos no Brasil. Rio de Janeiro: IBGE; 2010.
19. Santos RD, Gagliardi AC, Xavier HT, Magnoni CD, Cassani R, Lottenberg AM, et al. I Diretriz sobre o consumo de gorduras e saúde cardiovascular. Arq Bras Cardiol. 2013;100(1 Supl 3):1-40.

20. Francischi RP, Pereira LO, Freitas CS, Klopfer M, Santos RC, Vieira P, et al. Obesidade: atualização sobre sua etiologia, morbidade e tratamento. Rev Nutr. 2000;13:17-28.

21. World Health Organization (WHO). Obesity: prevention and managing the global epidemic: report of a WHO consultation. Geneva: WHO; 2000.

22. Cunha CM, Ferreira TC, Morales NM, Morales RR, Pinto RM, Silva $\mathrm{CH}$. Influência dos tipos de câncer pediátrico e das fases do tratamento na qualidade de vida dos cuidadores. Biosci J. 2013;29:774-80.

23. Given BA, Given CW, Sherwood P. The challenge of quality cancer care for family caregivers. Semin Oncol Nurs. 2012;28:205-12.

24. Ovayolu O, Ovayolu N, Tuna D, Serçe S, Sevinç A, Çöçelli LP. Quality of life of caregivers: a cross-sectional study. Int J Nurs Pract. 2014;20:424-32.

Recebido: Set 12, 2017 Aceito: Nov 26, 2017 